\title{
Deletion of SOCS7 leads to enhanced insulin action and enlarged islets of Langerhans
}

\author{
Alexander S. Banks, ${ }^{1}$ Jianze Li, ${ }^{2}$ Lisa McKeag, ${ }^{3}$ Marta L. Hribal, ${ }^{2}$ Masaki Kashiwada, ${ }^{3}$ \\ Domenico Accili, ${ }^{2}$ and Paul B. Rothman ${ }^{3}$
}

\begin{abstract}
1Department of Microbiology and 2Department of Medicine, College of Physicians and Surgeons, Columbia University, New York, New York, USA.
${ }^{3}$ Department of Internal Medicine, University of lowa, Roy J. and Lucille A. Carver College of Medicine, lowa City, Iowa, USA.
\end{abstract}

\begin{abstract}
NIDDM is characterized by progressive insulin resistance and the failure of insulin-producing pancreatic $\beta$ cells to compensate for this resistance. Hyperinsulinemia, inflammation, and prolonged activation of the insulin receptor (INSR) have been shown to induce insulin resistance by decreasing INSR substrate (IRS) protein levels. Here we describe a role for SOCS7 in regulating insulin signaling. Socs7-deficient mice exhibited lower glucose levels and prolonged hypoglycemia during an insulin tolerance test and increased glucose clearance in a glucose tolerance test. Six-month-old Socs7-deficient mice exhibited increased growth of pancreatic islets with mildly increased fasting insulin levels and hypoglycemia. These defects correlated with increased IRS protein levels and enhanced insulin action in cells lacking SOCS7. Additionally, SOCS7 associated with the INSR and IRS1 - molecules that are essential for normal regulation of insulin action. These data suggest that SOCS7 is a potent regulator of glucose homeostasis and insulin signaling.
\end{abstract}

\section{Introduction}

Insulin is the primary hormone that regulates glucose uptake in mammals. Resistance to insulin, as characterized by systemic diminution in insulin action, can occur spontaneously with age, obesity, or inflammation and is a fundamental component of NIDDM. Despite intensive study, the molecular mechanism underlying spontaneous insulin resistance is currently unknown.

The binding of insulin to the insulin receptor (INSR) induces its intrinsic tyrosine kinase activity, which results in the recruitment and phosphorylation of multiple substrates. Among these are the INSR substrate (IRS) proteins. Individual mutation of the Irs-encoding genes in mice has shown that each of these proteins has a unique role in insulin signaling. The IRS proteins are subject to several modes of negative regulation, presumably to ensure that INSR signaling is tightly controlled. For example, IRS protein stability is diminished in response to chronic cellular stimulation with insulin. This decrease is mediated by proteins that interact with IRS and initiate ubiquitin-mediated proteasomal degradation of IRS molecules (1-8). In addition, exposure of cells to lipopolysaccharide or the inflammatory cytokine TNF- $\alpha$ induces serine/threonine phosphorylation of IRS1, which results in decreased affinity for the INSR that correlates with reduced insulin signaling (9-12). Thus, inflammatory or chronic stimulation can provoke cellular insulin resistance via effects on IRS protein function $(1-3,5)$.

SOCS proteins are known to act as negative regulators of cytokine action via inhibition of JAK/STAT signaling (13). Recently, SOCS proteins have also been implicated in the negative regulation of insulin signaling $(14,15)$. The SOCS family consists of 8 proteins (cytokine-inducible SH2-containing protein [CIS] and SOCS1-SOCS7). Each protein contains a unique aminoterminal

Nonstandard abbreviations used: CIS, cytokine-inducible SH2-containing protein; GTT, glucose tolerance test; Hprt, hypoxanthine guanine phosphoribosyl transferase; INSR, insulin receptor; IRS, INSR substrate; ITT, insulin tolerance test; MEF, mouse embryonic fibroblast; Q-PCR, quantitative real-time PCR.

Conflict of interest: The authors have declared that no conflict of interest exists.

Citation for this article: J. Clin. Invest. 115:2462-2471 (2005).

doi:10.1172/JCI23853. domain of variable length, a central phosphotyrosine-binding SH2 domain, and a carboxyterminal SOCS box. Three mechanisms of SOCS action on JAK/STAT signaling have thus far been identified. SOCS proteins either directly bind to and inactivate tyrosine kinases, bind to cytokine receptors and occupy docking sites otherwise available for signal transduction mediators such as STATs and SHP2, or function as adapters by forming a complex with elongins $\mathrm{B} / \mathrm{C}$ and facilitating the ubiquitination of signaling proteins and their subsequent proteasomal degradation (16). Among the SOCS proteins, SOCS7 is unique in that it contains 4 polyproline regions that may allow association with $\mathrm{SH} 3$ domain-containing proteins. SOCS7 interacting molecules have been identified in 2 independent screens utilizing the yeast 2-hybrid and coimmunoprecipitation assays. In these experiments, association was established with the signaling molecules Grb-2 and Stat-3; the tyrosine kinases Egfr, Lck, and Hck; the lipase PLC- $\gamma$; and the multiple SH3 domain-containing protein Vinexin. Both studies found interactions with the adaptor molecule Nck $(17,18)$. In addition, a glutathione-S-transferase-fusion protein of the SOCS7 SH2 domain was found by mass spectrometry to associate with IRS4 and p85, the regulatory subunit of PI3K (19). As a majority of these proteins act in the insulin-signaling pathway, these data suggest an interaction between SOCS7 and the insulin-signaling cascade.

There is a growing body of evidence supporting a role for the SOCS proteins in insulin signaling. Socs genes are induced by insulin treatment in a number of tissues and cell lines (18, 20-22). SOCS1 and SOCS3 interact with INSR, IRS1, and IRS2 $(6,21,23-25)$, and forced expression of either SOCS protein in the liver induces insulin resistance. In cultured cells, overexpression of SOCS1 or SOCS3 has multiple effects, including reduced IRS protein levels, decreased Akt/PKB phosphorylation, reduced IRS-associated p85 ${ }^{\text {PI3K }}$ levels, and decreased PI3K activity $(6,21$, $22,25,26)$. In cells treated with antisense knockdown vectors for Socs 1 and Socs 3 or in Socs $3^{-/-}$adipocytes, TNF- $\alpha$ fails to generate insulin resistance. In vitro studies suggest that SOCS1 causes insulin resistance, and the reported hypoglycemia in Socs $1^{-/-}$mice appears to confirm these data. However, these results may be con- 
founded by the fact that Socs $1^{-/-}$mice suffer from severe cachexia and premature death due to dysregulated IFN- $\gamma$ expression. More significantly, Socs $1^{-/-}$mouse embryonic fibroblast (MEF) cells exhibit increased adipocyte differentiation, which is a measure of sensitivity to insulin (23). Conversely, enforced adenoviral-driven Socs 1 expression in mouse liver leads to hyperglycemia, hyperinsulinemia, and resistance to insulin injections, with dramatic reductions in hepatic IRS1 and IRS2 levels (6).

Until very recently most studies have focused on the actions of CIS, SOCS1, SOCS2 and SOCS3; little has been known about the function of other SOCS proteins. It now appears that these relatively obscure proteins are the evolutionary predecessors to the SOCS family. The sequencing of the Drosophila genome revealed 3 Socs genes, 1 homologous to Socs 4 and Socs 5 and 2 homologous to Socs 6 and Socs7. The single Socs gene identified in the Caenorhabditis elegans genome sequence is similar to Socs6 and Socs7. This finding supports a conserved function for these proteins in animal biology, possibly beyond JAK/STAT signaling $(16,27)$. Examination of the murine SOCS6 and SOCS7 proteins has shown specific binding affinity for IRS4 and P85 ${ }^{\mathrm{PI} 3 \mathrm{~K}}$ and interaction of SOCS7 with IRS2 (19), which suggests that they may affect insulin-signaling molecules. Several pieces of evidence suggest that SOCS6 regulates insulin action; however, the nature of this effect is uncertain. SOCS6 associates with the INSR and inhibits signal transduction when ectopically expressed in rat hepatoma cells (24). However, mice deficient in Socs6 do not exhibit any obvious defects in glucose homeostasis (19). It was therefore somewhat unexpected that global overexpression of Socs 6 in transgenic mice caused increased glucose tolerance and insulin signaling through decreased $\mathrm{p} 85^{\text {PI3K }}$ levels (28).

SOCS7 protein shares greater than 50\% identity with SOCS6 in both the SH2 domain and the SOCS box. Thus, the function of SOCS7 may overlap with that of SOCS6 sufficiently so that SOCS7 is able to compensate for SOCS6 deficiency. To clarify the physiological role of SOCS7 in vivo, we generated mice with a targeted disruption of the Socs7 gene. Our data demonstrate that mice deficient for the Socs 7 gene show increased sensitivity to insulin via a mechanism that may involve increased accumulation and activity of IRS1.

\section{Results}

Expression of Socs 7 is upregulated by insulin. To determine the expression pattern of Socs7, mRNA levels in tissues from 3-7 female C57BL/6J (C57BL) and 129S6/SvEvTac mice (129S6) were quantified using a quantitative real-time PCR (Q-PCR) assay. In C57BL mice, Socs 7 is expressed at the highest levels in isolated pancreatic islets, whole brain, and skeletal muscle, while lower levels are observed in liver, whole pancreas, perigonadal fat, skin, and spleen relative to levels of bypoxanthine guanine phosphoribosyl transferase (Hprt) mRNA (Figure 1A). A similar Socs7 expression pattern is seen in 129S6 mice, with notable differences in whole brain (decreased 5-fold), spleen (increased 6-fold), and isolated pancreatic islets (decreased 2,000-fold). To validate our Q-PCR data, a 129S6 murine multiple-tissue Northern blot was probed with a Socs7 cDNA. As shown in Figure 1B, Socs7 is expressed at high levels in skeletal muscle, brain, kidney, and testis and at low levels in other tissues. The larger transcript corresponds to the predicted size of the mRNA encoding Socs 7 and is consistent with our Q-PCR data and a recently reported Northern blot (29). The small hybridizing mRNA (which predominates in the testis) is of unclear significance but is consistent with a previously reported tissue distribution of Socs7 using polyA RNA (19). The expression pattern of Socs 7 is dramatically different from that reported for Socs6, which, although more widely expressed, is found only at low levels in muscle and brain (19).

If SOCS7 were to act in a classical negative-feedback loop on insulin signaling, Socs 7 message would be induced following insulin stimulation. We therefore examined the transcriptional response of Socs 71 hour after a physiological dose $(0.75 \mathrm{U} / \mathrm{kg})$ of insulin in C57BL and 129S6 mice. Induction of Socs 7 mRNA levels differed between the 2 strains. In muscle, the primary site of insulin-stimulated glucose utilization in mammals, Socs 7 mRNA levels increased more than 4-fold in C57BL mice but decreased by a third in 129S6 muscle. An inverse pattern is observed in mRNA from whole brain, with a 2.5 -fold induction in $129 \mathrm{~S} 6$ and a reduction by half in C57BL mice. In addition, Socs7 message was induced 2-fold in liver from $129 \mathrm{~S} 6$ mice but was unchanged in C57BL mice. We observed no significant transcriptional change in pancreas, fat, or spleen from either strain (Figure 1C). This result demonstrates that Socs 7 expression is also rapidly regulated by insulin action in tissues that are important for regulation of glucose and insulin secretion in a strain-dependent manner. Previously, insulin was reported to regulate expression of other Socs genes in myoblasts, myotubes, and adipocytes $(21,25,26,30)$. These data suggest that SOCS proteins are variably regulated both in quantity and tissue distribution and may play complementary roles in regulation of insulin signaling.

SOCS7 interacts with multiple components of the insulin-signaling pathway. To help define the molecular mechanism by which SOCS7 participates in insulin signaling, we examined the interactions of SOCS7 with proximal mediators of insulin signaling in transfected HEK293 cells. cDNAs for Insr or Irs1 were expressed individually or were coexpressed with epitope-tagged Socs7. Lysates from these cells were subjected to immunoprecipitations performed with antibodies recognizing INSR or IRS1 (Figure 1D). SOCS7 coimmunoprecipitates when coexpressed with IRS1. In addition, SOCS7 coimmunoprecipitates with overexpressed INSR; however, SOCS7 is also capable of coimmunoprecipitating with the endogenous INSR. IRS1 and INSR both failed to coimmunoprecipitate with a control protein, Xpress epitope-tagged LacZ (data not shown). Although some proteins interact with IRS1 in a phosphorylation-dependent manner, this was not the case for SOCS7. Phosphatase treatment did not abrogate the interaction of SOCS7 with IRS1 (data not shown). These results raise the possibility that SOCS7 may interact with IRS1 in a manner only partially dependent on phosphorylation and may play a role in regulating activation or stability of the INSR or IRS proteins under basal and activated conditions.

Generation of Socs7-deficient mice reveals strain-dependent perinatal lethality. To investigate the in vivo function of Socs7, gene targeting was performed to generate Socs7-deficient mice (Figure 2A and Methods). Socs $7^{+/-}$mice were intercrossed, and genotypes were determined by PCR (Figure 2C) and confirmed by Southern blot (Figure 2B). Functional disruption of the Socs 7 gene was confirmed by Northern blot analysis of mRNA from liver, skeletal muscle, and testis. Although expression of the full-length Socs 7 transcript is abrogated in liver and skeletal muscle, low levels of Socs 7 expression persist in the testis. Additionally, the testis-specific isoform of Socs 7 was not deleted in the targeted mice. This implies that the smaller transcript is not the product of exons 3-6 deleted in the targeted mice (Figure 2D). Socs $7^{+/-}$mice were initially obtained in a mixed 129S6 × C57BL genetic background and were subsequently 

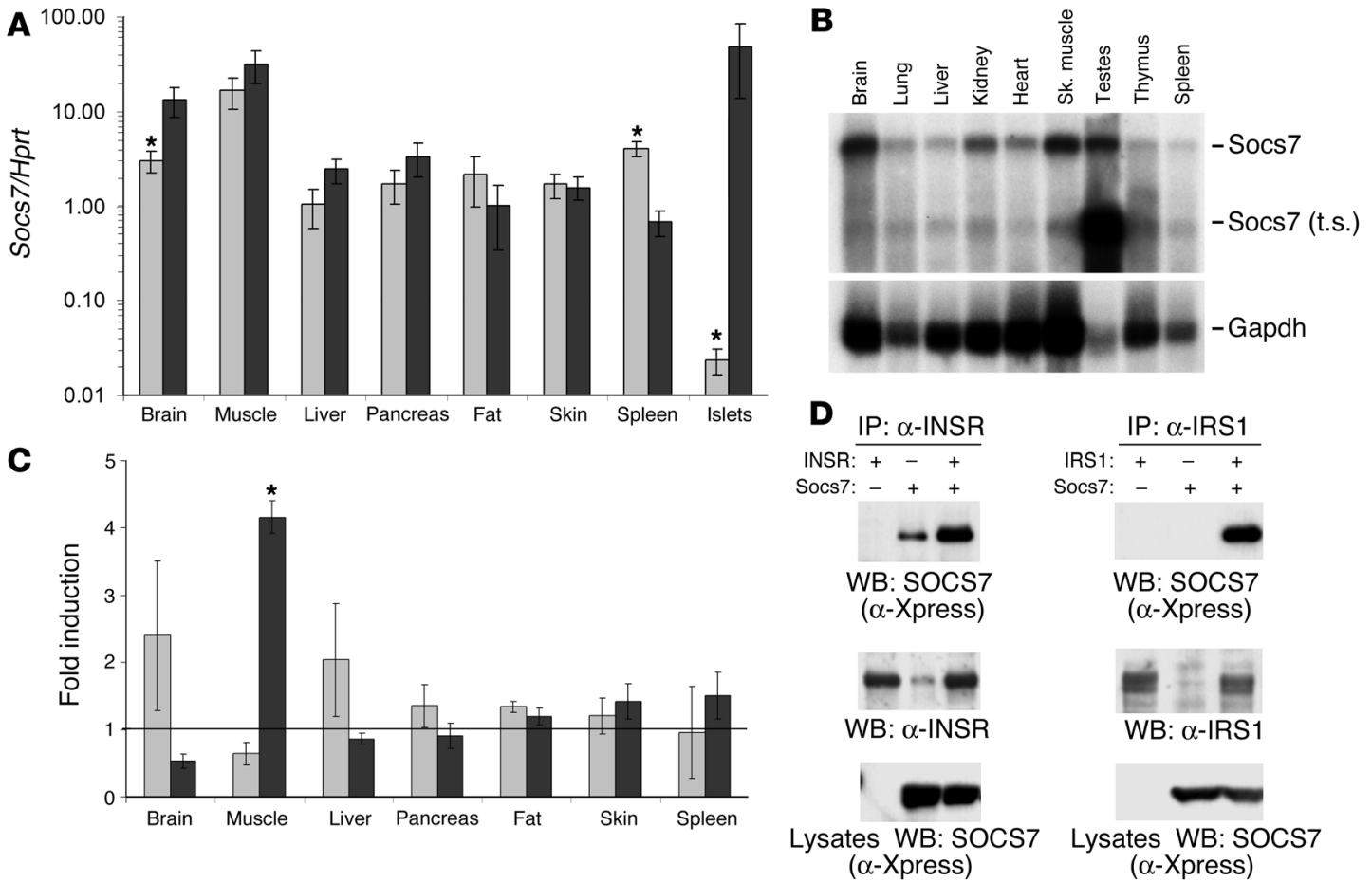

Figure 1

Socs7 expression patterns, induction, and protein associations. (A) Socs7 mRNA expression was determined by Q-PCR in 129S6 and C57BL mice fed a standard $10 \%$ fat diet ad libitum. Values are normalized relative to Hprt mRNA and plotted on a log scale. In addition to whole mouse tissues, collagenase-purified islets were also examined. Results are representative of 3 to 7 mice; error bars indicate \pm SEM. ${ }^{*} P<0.05$ between genotypes (1-tailed Student's $t$ test). 129S6 and C57BL/6 are indicated by light gray and dark gray bars, respectively. (B) A murine $129 \mathrm{~S} 6$ multiple-tissue Northern blot was generated and probed with Socs7 full-length cDNA. Two bands were observed, the full-length transcript and a smaller, testis-specific (t.s.) isoform. The blot was stripped and reprobed with a Gapdh cDNA fragment as a loading control. Sk., skeletal. (C) Induction of Socs7 mRNA by insulin. Q-PCR for Socs7 in tissues isolated 1 hour after a physiological dose $(0.75 \mathrm{U} / \mathrm{kg})$ of insulin. Values are given as fold induction over level of Socs7 mRNA expression in untreated mice. Results from 4 treated (dark gray bars) and 4 untreated mice (light gray bars) are included. ${ }^{*} P<0.05$ between stimulated and unstimulated mice of the same genotype (1-tailed Student's $t$ test). (D) Association of SOCS7 with insulin-signaling molecules. A full-length Socs7 cDNA was tagged with Xpress epitope and transfected into HEK293 cells with INSR or IRS1 as indicated. Immunoprecipitates of INSR or IRS1 and whole-cell lysates were blotted with an antibody recognizing SOCS7. Blots were stripped and reprobed with either antibodies against INSR or IRS1. WB, Western blot.

backcrossed 7 generations into C57BL/6J. Regardless of genetic background, Socs $7^{-1-}$ mice were born at the predicted Mendelian frequency $\left(n=258 ; \operatorname{Socs} 7^{+/+}, 22 \%\right.$; Socs $7^{+/-}, 53 \%$; Socs $\left.7^{-/-}, 25 \%\right)$. Mixed background $\operatorname{Socs} 7^{-1-}$ mice were identical in length, weight, and body composition to wild-type littermates at up to 6 months of age (data not shown). However, Socs $7^{-/-}$mice on the C57BL background displayed multiple defects at an early age. More than half of all C57BL Socs $7^{-1-}$ mice had severe hydrocephalus and growth retardation concomitant with hypoglycemia that resulted in perinatal lethality (29) (Figure 2E and data not shown). Because of these defects, subsequent analysis was performed using mice on the mixed background. Thus, Socs 7 deficiency causes a dramatic phenotype that is strongly influenced by genetic background.

Altered glucose homeostasis in Socs7-deficient mice. IRS proteins are essential mediators of insulin action. The interaction of SOCS7 with these proteins suggested that mice deficient in Socs 7 might have altered glucose homeostasis. To evaluate this possibility, blood glucose and plasma insulin concentrations of overnightfasted Socs $7^{-1-}$ and wild-type mice were determined. No differences were observed in mice aged 6-8 weeks. However, evaluation of mice between 5 and 8 months of age showed that wild-type mice developed mild hyperglycemia while, in contrast, glucose levels of the older Socs $7^{-/-}$mice remained normal (Figure $3 \mathrm{~A}$ and data not shown). On the other hand, insulin levels of wild-type mice were similar to those of young mice, but older Socs $7^{-1-}$ mice developed a small but significant hyperinsulinemia. These results may be indicative of a subtle alteration in glucose homeostasis.

To further analyze glucose tolerance and insulin sensitivity, mice were subjected to glucose tolerance tests (GTTs) and insulin tolerance tests (ITTs). Following intraperitoneal glucose administration, 2- to 8-month-old Socs $7^{-1-}$ mice cleared glucose more rapidly than control animals and thus exhibited improved glucose tolerance (Figure 3B, upper panels). These results could not be explained by differences in insulin release following the glucose challenge (data not shown). Another possible basis for the improved glucose tolerance of the Socs $7^{-/-}$mice is an increased sensitivity of target organs to insulin. To test this hypothesis, ITTs were performed. Consistent with the GTT results, Socs $7^{-/-}$mice exhibited increased insulin sensitivity compared with wild-type mice (Figure 3B, lower panels). Notably, following insulin administration, blood glucose levels in the Socs $7^{-1-}$ mice dropped to levels lower than those of wild-type littermate controls. Furthermore, unlike glucose levels in wild-type mice, those in Socs $7^{-1-}$ did not return to basal levels for up to 2 hours after administration 
A
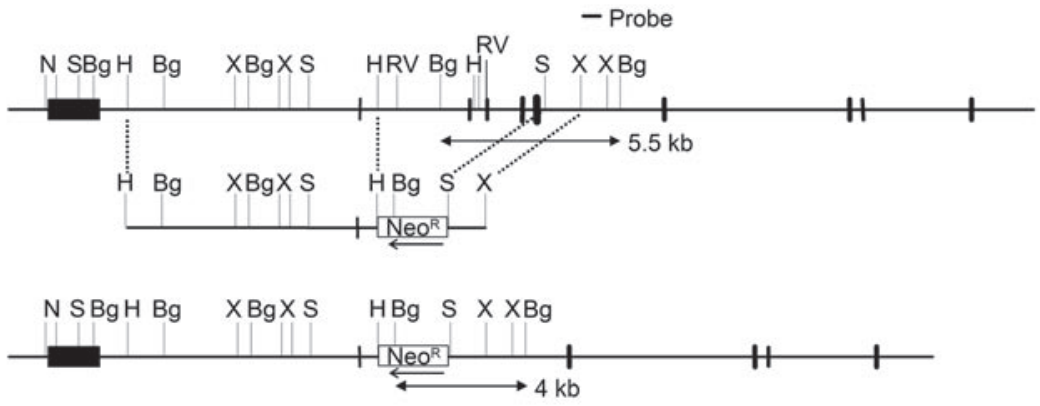

B

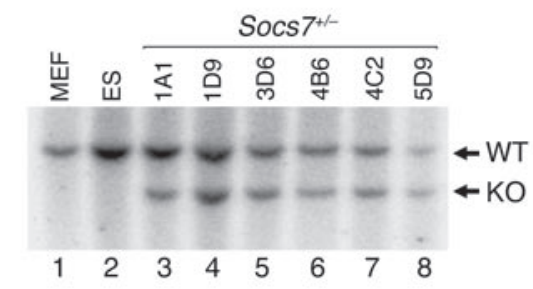

D

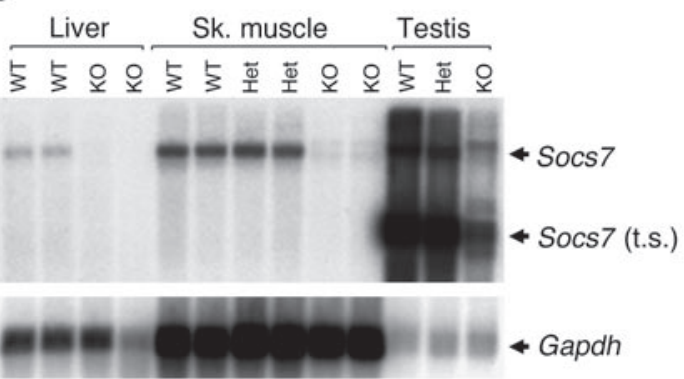

Figure 2

Targeted disruption of the Socs7 gene by homologous recombination. (A) Schematic representation of mouse Socs7 gene (top), targeting construct (middle), and targeted allele (bottom). Relevant restriction sites are indicated: Bg, Bglll; H, HindIII; N, Notl; S, Sall; X, Xbal. The black boxes indicate exons. $\mathrm{Neo}^{\mathrm{R}}$ refers to the positive selection marker. The genomic fragment used as a probe for Southern blot analysis and the expected fragments after Bglll digestion are indicated. (B) Southern blot analysis of Bglll-digested genomic DNA from ES cell clones. The blot was hybridized with the indicated $3^{\prime}$ external probe. Lanes 1 and 2 show the wild-type allele from MEF and ES cells. Lanes 3-8 are from ES cells with correctly targeted alleles. (C) PCR analysis of genomic DNA from the tails of wild-type, heterozygous (Het), and knockout littermates. (D) Northern blot analysis of liver, skeletal muscle, and testis from wild-type, heterozygous, and knockout mice, showing the absence of Socs7 full-length transcript expression. (E) Growth retardation and hydrocephalus in Socs7 ${ }^{-/-}$ C57BL/6 mice. At 20 days of age, affected knockout mice exhibited a $40 \%$ decrease in weight when compared with heterozygote littermates on the C57BL/6 background (upper panel). Severe hydrocephalus is also present in a 4-week-old Socs $7^{-/-}$mouse (lower panels). Hydrocephalus was not apparent in the mixed-background mice used in this study (image not shown).

of insulin. One possible explanation for this result is a defective counterregulatory response to hypoglycemia, such as decreased glucagon secretion. To evaluate this possibility, we examined glucagon levels after insulin administration and found that Socs $7^{-1-}$ mice had the same glucagon levels as wild-type mice (data not shown). Thus, systemic insulin-induced hypoglycemia does not appear to be caused by altered glucagon secretion; instead, it appears to reflect heightened sensitivity to insulin.

Pancreatic islets in Socs $7^{-1-}$ mice become progressively enlarged. Sensitivity to insulin and adequate insulin secretion are essential for normal glucose homeostasis. Socs $7^{-/}$mice exhibited reduced glycemia during a GTT, suggesting an exaggerated insulin response to the glycemic load. To explore this possibility, we evaluated islets and insulin content of 5- to 8-monthold mice. Fixed and embedded sections from the pancreata of wild-type and $\operatorname{Socs} 7^{-1-}$ mice were stained with anti-insulin or antiglucagon antisera. Analysis revealed islet number to be increased 1.8-fold in Socs $7^{-1-}$ animals. The mean and median islet sizes in organs from Socs $7^{-/-}$mice were increased 1.9-fold and 1.7-fold, respectively, relative to those in littermate controls. However, the most striking difference was a 5 -fold increase in the percentage of large islets (> 750,000 $\mu^{3}$; Figure $\left.3 \mathrm{C}\right)$. The same evaluation was performed in mice at 3 weeks of age; although islet number was modestly increased in Socs $7^{-/-}$mice in this group, there was no significant difference in islet size, proliferation, or apoptosis as measured by BrdU incorporation or staining for cleaved caspase-3 (data not shown). These data suggest that SOCS7 has an important role in the regulation of islet mass in adult mice. Furthermore, most instances of islet hyperplasia are associated with insulin resistance. Paradoxically, in the case of Socs 7 deficiency, we observed increased islet size in mice that are more sensitive to insulin.

Altered IRS activation in Socs7-deficient skeletal muscle. Skeletal muscle is the site of the greatest insulin-induced glucose uptake. It is also the whole tissue with highest expression of Socs 7 mRNA. To investigate mechanisms responsible for the increased insulin sensitivity of Socs $7^{-1-}$ mice observed in response to an ITT, tyrosine phosphorylation of INSR and IRS1 proteins were examined in skeletal muscle after bolus insulin injection into the inferior vena cava. Tyrosine phosphorylation of immunoprecipitated IRS1, IRS2, and INSR was not increased following insulin stimulation, nor were the levels of phosphoserine Akt altered relative to those of wild-type controls (Figure 4A and data not shown). However, an electrophoretic mobility shift for IRS1 consistent with a covalent posttranslational modification was noted under both resting and insulin-stimulated conditions in Socs $7^{-1-}$ muscle. Although the nature of this modification has not been determined, several possible posttranslational modifications to IRS have been reported, including serine/threonine phosphorylation, glycosylation, and ubiquitination. These data, along with the GTT and ITT experiments, suggest that the response of Socs7-deficient mice to insulin is enhanced - possibly through the regulation of IRS1.

Protein degradation of IRS1 by SOCS7 in vitro. Multiple studies have proposed a molecular mechanism of insulin resistance due to 

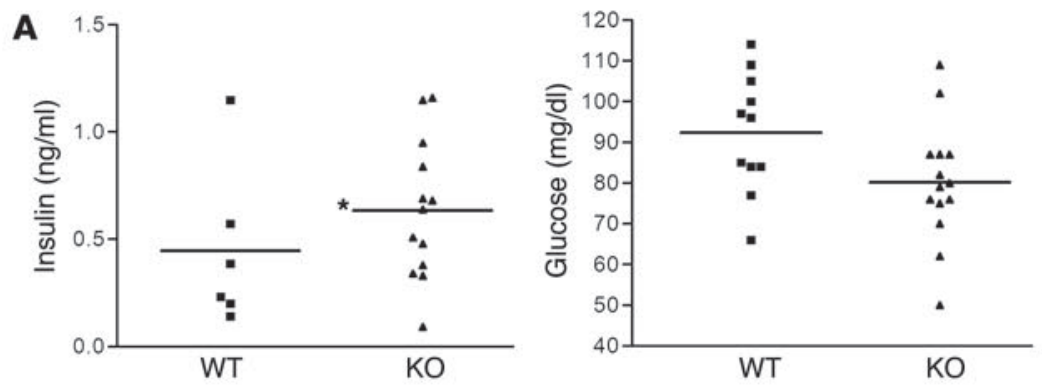

B
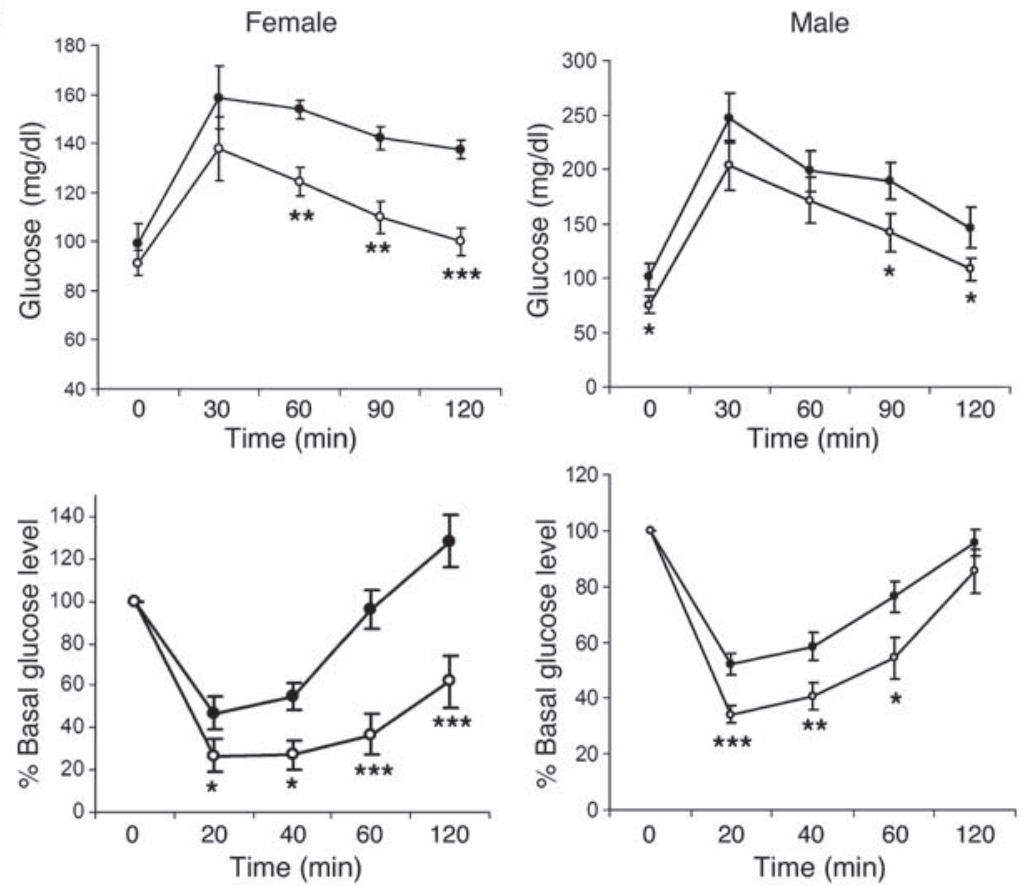

\section{Figure 3}

Increased insulin sensitivity in Socs7 $7^{-/-}$ mice. (A) Blood glucose (right) and plasma insulin (left) concentrations in overnight-fasted wild-type and Socs $7^{-/-}$mice aged at least 6 months. (B) Prolonged hypoglycemia in Socs7-/- mice following ITT and increased glucose clearance in GTT. All values are expressed as the mean \pm SEM obtained from wild-type and Socs $7^{-1-}$ mice. All tests were performed on 5- to 8-month-old mice. Filled circles, wildtype mice; open circles, Socs7-/- mice. ${ }^{\star} P<0.05 ;{ }^{* *} P<0.01 ;{ }^{* *} P<0.001$ (1-tailed Student's $t$ test). For ITT, results are given as a percentage of basal glucose concentration. (C) Increased islet size and $\beta$ cell mass in Socs $7^{-/-}$mice compared with wild-type littermates as assayed by staining with anti-insulin (upper panels) and antiglucagon antibodies (lower panels). Histogram represents the quantization of islet size performed using SPOT imaging software. Black bars, wild-type mice; white bars, Socs7 $7^{-/}$mice. Magnification, $\times 10$.
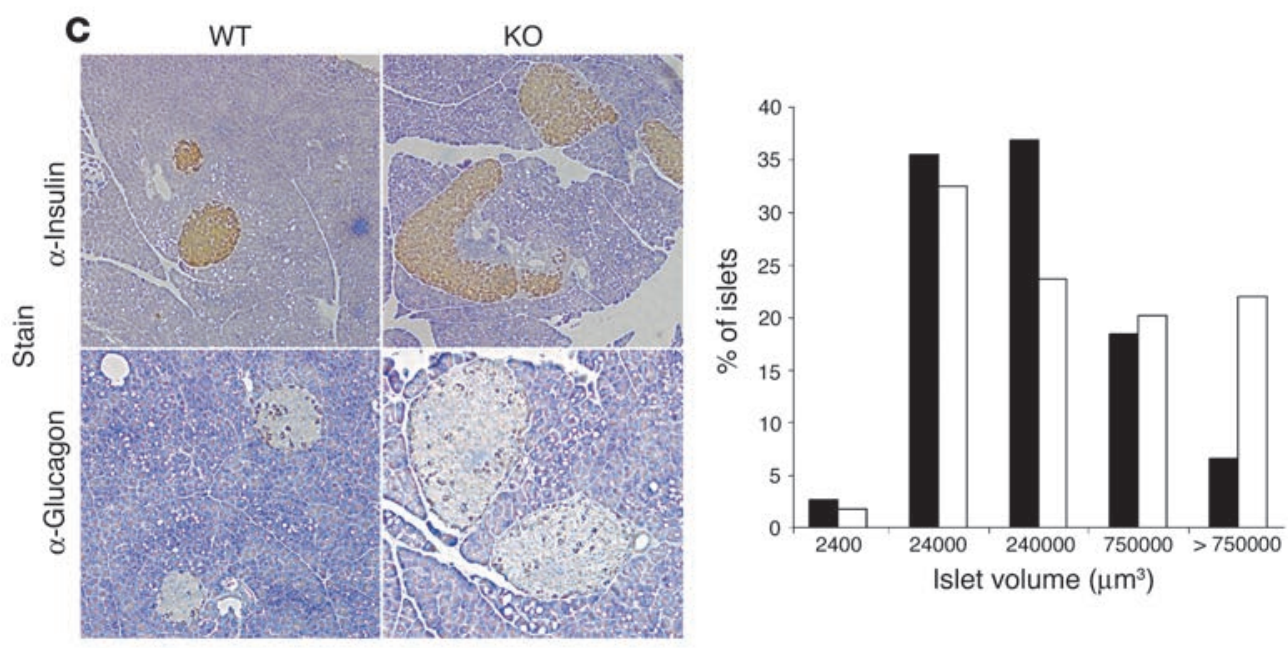

increased proteasomal degradation of IRS1 or IRS2 in response to cytokines or insulin $(1-3,5,6,9)$. As SOCS proteins are implicated in targeting proteins for ubiquitination and proteasomal degradation, we hypothesized that SOCS7 may regulate IRS1 protein stability. To examine the effects of SOCS7 activity, we constructed 3 SOCS7 mutants (Figure 4B). These proteins lacked the $\mathrm{N}$-terminal polyproline domains $(\Delta N)$, the C-terminal SOCS box $(\Delta S B)$, or a point mutation in the phosphotyrosine-binding site of the $\mathrm{SH} 2$ domain $(\mathrm{R} \rightarrow \mathrm{K})$. When an Irs1 cDNA was transfected into HEK293 cells either alone or with a cDNA for ubiquitin, robust protein expression was detected (Figure 4C, lanes 2 and 4). However, addition of wild-type or an $\mathrm{N}$-terminal deletion $(\Delta \mathrm{N})$ of SOCS7 resulted in dramatic reduction of IRS1 protein levels (Figure 4C, lanes 6 and 7, respectively). IRS1 protein levels were no longer reduced 
A

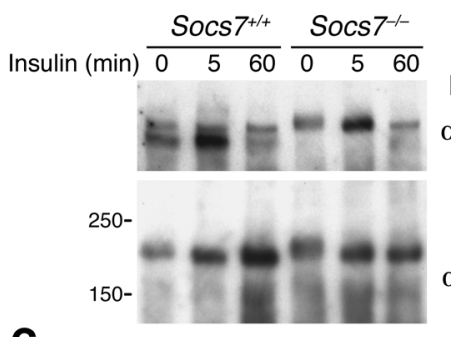

B

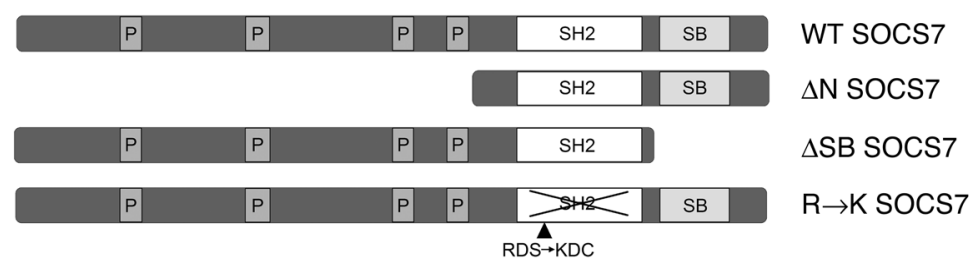

D

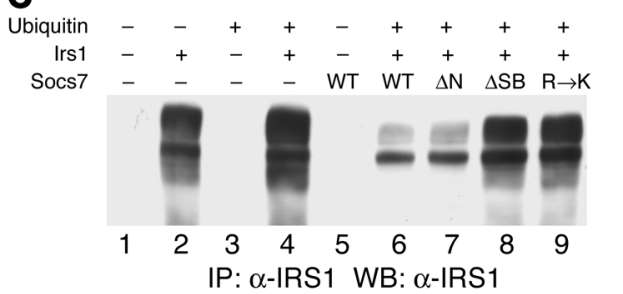

Blot:
$\alpha-p$ Tyr $\alpha-\operatorname{IRS} 1$

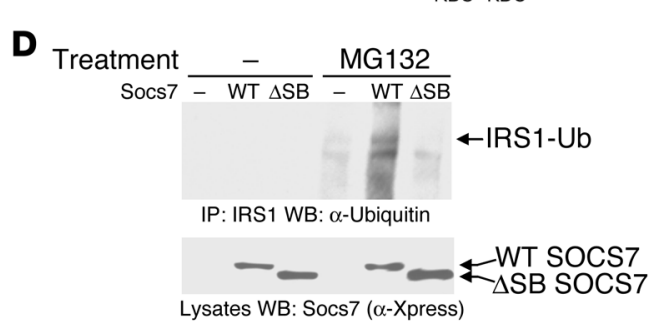

Socs7 $\frac{-}{-W T \Delta S B} \frac{M G 132}{- \text { WT } \Delta S B}$

\section{Figure 4}

Socs7 affects insulin signaling and IRS1 protein stability. (A) Insulin stimulation of wild-type or Socs7-/- mice. Six-week-old female mice were injected with a 5-unit bolus of human insulin. Soleus muscle was harvested at the times indicated. IRS1 tyrosine phosphorylation ( $p$-Tyr) was assayed by immunoprecipitation and immunoblot. The blots were stripped and reprobed for IRS1 to assess protein loading. Relative molecular weight markers are indicated in $\mathrm{kDa}$. (B) Schematic representation of SOCS7 mutants. Wild-type SOCS7 contains 4 polyproline domains (P), an $\mathrm{SH} 2$ domain, and a SOCS box domain (SB). The $\Delta \mathrm{N}$ mutant lacks the $4 \mathrm{~N}$-terminal polyproline domains. The $\Delta \mathrm{SB}$ mutant contains a deletion of the C-terminal SOCS box. The $\mathrm{R} \rightarrow \mathrm{K}$ mutation includes 2 point mutations in the phosphotyrosine-binding domain of the $\mathrm{SH} 2$ domain. (C) HEK293 cells were transfected with cDNAs for ubiquitin, Irs1, and wild-type or mutant Socs7 as indicated. Forty-eight hours after transfection, cells were harvested, and immunoprecipitation and immunoblotting for IRS1 were performed. (D) HEK293 cells were transfected as in C. Then, 42 hours after transfection, cells were preincubated for 30 minutes with either $20 \mu \mathrm{M}$ of the proteasome inhibitor MG132 (right 3 lanes) or methanol carrier as a control. Cells were then stimulated with $100 \mathrm{nM}$ insulin for 6 hours before harvest and immunoprecipitation with IRS1 and immunoblot with anti-ubiquitin antibody. Whole cell lysates were blotted with an antibody recognizing SOCS7. IRS1-Ub, ubiquitinated IRS1.

when cotransfected with a SOCS7 protein without the SOCS box $(\triangle \mathrm{SB})$, a domain implicated in ubiquitin conjugation (Figure 4C, lane 8). Similarly, SOCS7 containing a point mutation in the phosphotyrosine-binding site of the $\mathrm{SH} 2$ domain $(\mathrm{R} \rightarrow \mathrm{K})$ failed to reduce IRS1 protein levels (Figure 4C, lane 9). The SOCS box is an approximately 40 -amino acid region postulated to recruit the elongin $\mathrm{B} / \mathrm{C}$ ubiquitin ligase machinery. The $\mathrm{SH} 2$ domain of SOCS proteins determines phosphotyrosine binding specificity and thereby targets phosphorylated proteins for ubiquitination. The failure of the SH2 domain and SOCS box mutants to reduce IRS1 protein levels suggests that wild-type SOCS7 may decrease the protein stability of IRS1 by ubiquitination.

IRS1 ubiquitination was examined to investigate the mechanism of reduced IRS1 protein levels when cotransfected with wild-type but not $\triangle$ SB SOCS7. If IRS1 is being targeted for proteasomal degradation by wild-type SOCS7, inhibiting the proteasome should result in an accumulation of ubiquitinated IRS1. Without the proteasome inhibitor MG132, IRS1 ubiquitination was almost undetectable (Figure 4D). However, after MG132 treatment, a baseline amount of ubiquitinated IRS1 was observed in cells without exogenous SOCS7. The amount of ubiquitinated IRS1 was enhanced in cells cotransfected with wild-type but not with $\triangle$ SB SOCS7. These results suggest that the diminished IRS1 protein levels observed in Figure $4 \mathrm{C}$ are the result of ubiquitination and proteasome-mediated degradation caused by SOCS7.

Enhanced insulin signaling and insulin-mediated adipogenesis in Socs $7^{-1-}$ fibroblasts. As SOCS7 is capable of reducing IRS1 protein levels in vitro and Socs $7^{-1-}$ mice demonstrate increased insulin sensitivity, we hypothesized that Socs $7^{-1-}$ mice might have lower rates of protein turnover and thus higher steady-state levels of IRS proteins. Therefore, IRS1 protein levels were examined in Socs $7^{-/-}$cells following prolonged stimulation with IGF-1. Six hours after exposure to IGF-1, IRS1 protein levels were increased in Socs $7^{-/}$MEF cells compared with cells derived from Socs $7^{+/+}$mice (Figure 5A, lanes 5 and 6). The diminished protein levels in wild-type cells were blocked using lactacystin, a proteasome inhibitor (Figure 5A, lanes 7 and 8). The increased levels of IRS1 in Socs7-deficient cells suggest that Socs7 is required for stimulation-dependent protein degradation.

Adipocyte differentiation in vitro is an insulin-dependent process. Disruption of essential insulin-signaling molecules such as Insr, Irs $1, \mathrm{Akt} 1 / 2$, and constitutively active Foxo 1 has been shown to reduce adipogenesis (31-33). Inversely, disruption of Socs1 (an inhibitor of insulin action), exogenous expression of the PI3K substrate (Akt1), or a dominant-negative insulindependent transcription factor (Foxo1) all result in increased adipocyte formation $(23,33,34)$. To address whether insulin signaling was altered in Socs $7^{-/-}$fibroblasts, we examined the ability of insulin to induce the differentiation of these cells into adipocytes. Cells from wild-type, Socs1-, Socs3-, and Socs7-deficient embryos were allowed to differentiate for 14 days and either harvested for RNA or stained with the lipid-soluble dye, oil red $\mathrm{O}$. Both Socs $7^{-/-}$and Socs $1^{-/-}$MEF cells showed increased adipocyte differentiation as determined by oil red $\mathrm{O}$ staining, but this was more dramatic in Socs $7^{-/-}$MEF cells (Figure $5 \mathrm{~B}$ ). These data were confirmed with Q-PCR analysis of the adipose differentiationinduced genes Pparg and Glut4. Differentiation of 3T3-L1 preadipocytes sharply increased expression of these genes. Consistent with this result is the elevated expression of Pparg and Glut4 in 
A

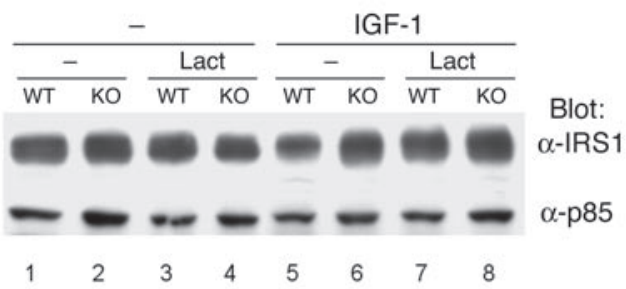

B

WT

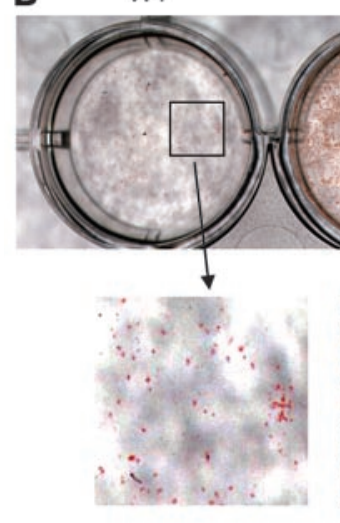

Socs $7^{-1-}$

$\operatorname{Socs} 1^{-1-}$

$\operatorname{Socs} 3^{-1-}$

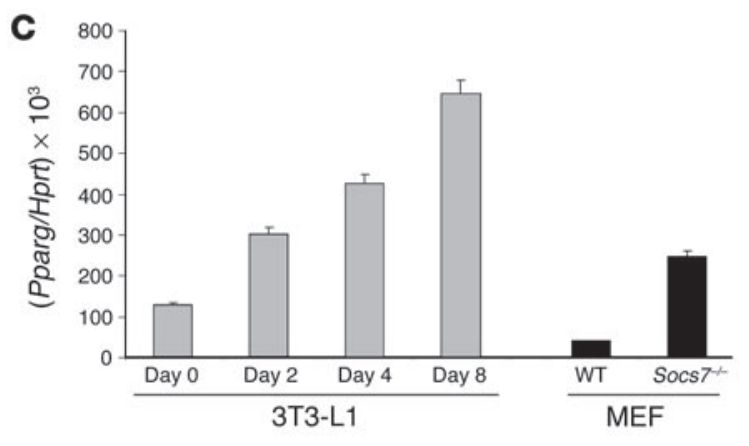

Socs $7^{-/-}$MEF cells (Figure 5C and data not shown). Results are representative of 3 to 5 different clonal isolates of MEF cells. As the differentiation protocol required a prolonged insulin treatment and we have demonstrated that Socs $7^{-/-}$cells are resistant to insulin-induced IRS1 degradation (Figure 5A), the enhanced adipogenesis may be due to enhanced insulin action. Furthermore, these data support an important role for Socs 7 in regulation of insulin-dependent adipogenesis.

\section{Discussion}

Socs 7 mRNA is highly expressed in organs that are essential for regulation of glucose homeostasis, and it is regulated by insulin in these tissues. SOCS7 protein associates with the proximal signaling molecules in the INSR pathway, and mice or cells lacking this protein display physiological signs of heightened sensitivity to insulin. Thus, SOCS7 appears to have an important role in the regulation of INSR signaling. SOCS7 is evolutionarily conserved. The SOCS6/SOCS7 precursor found in C. elegans is the evolutionary precursor to the mammalian SOCS family (27). Although the C. elegans SOCS protein lacks the $\mathrm{N}$-terminal domain unique to other SOCS proteins, our data utilizing the human SOCS7 $\Delta \mathrm{N}$ mutant demonstrates that this region may be dispensable. We hypothesize that manipulation of SOCS7 in model organisms such as C. elegans, Drosophila, and zebrafish will help to fully define

\section{Figure 5}

Resistance to stimulation-induced IRS1 degradation and increased adipogenesis in Socs7-deficient cells. (A) Socs7+/+ (WT) or Socs7 $7^{-/-}$(KO) MEF cells were serum starved for 14-16 hours before a 30-minute pretreatment without (lanes 1, 2, 5, and 6) or with (lanes 3, 4, 7, and 8) lactacystin (Lact), a proteasome inhibitor. Cells were then stimulated with $10 \mathrm{nM}$ IGF-1 for 6 hours. Immunoblotting for IRS1 was performed, followed by membrane stripping and reprobing with anti-p85 as a protein loading control. (B) Wild-type and Socs7-, Socs 1-, and Socs3-deficient MEF cells were subjected to adipocyte differentiation (see Methods). Differentiation was scored either by staining for oil red $\mathrm{O}$ to measure triglyceride accumulation or by (C) Q-PCR for Pparg (normalized to Hprt) relative to 3T3-L1 adipocytes during differentiation.

roles in development, glucose regulation, and signaling. In addition, human and murine SOCS7 proteins share greater than $95 \%$ sequence identity. In our assays, we determined that overexpression of SOCS7 can cause decreased protein levels of IRS1, which is commonly found in human obesity and type 2 diabetes $(35,36)$. It will therefore be of great interest to determine whether SOCS7 is abnormally regulated in patients with obesity or type 2 diabetes.

Defining the specificity of SOCS proteins requires multiple approaches. Much of the literature describing regulation of insulin signaling by SOCS molecules relies on exogenous gene expression to determine potential function. While these studies have been crucial to furthering our understanding of SOCS protein function, there are examples in which high-level SOCS protein expression, which is normally tightly controlled, causes effects on proteins or signaling that are not bona fide physiological targets. Accordingly, in vivo models have proven an indispensable complement to these studies. Both transgenic overexpression and gene ablation studies have helped in discriminating which pathways are regulated by SOCS proteins. However, even in vivo studies have their limitations and have generated seemingly intractable discrepancies. Transgenic overexpression of CIS strongly suggests a role for this protein in the inhibition of STAT5A/B, as the phenotype of deficient lactation and reduced IL-2, IL-3, and EPO responses resembles that of STAT5-deficient mice. However, CIS-deficient mice exhibit no detectable abnormalities (reviewed in ref. 37). A similar paradigm exists for the role of SOCS6 in the regulation of insulin signaling. Global overexpression of Socs 6 causes increased insulin sensitivity and glucose tolerance (28). This phenotype is similar to that of p $85^{\mathrm{PI} 3 \mathrm{~K}}$ heterozygous mice, implicating $\mathrm{p} 85^{\mathrm{PI} 3 \mathrm{~K}}$ as a regulatory target of SOCS6 (38). Yet Socs $6^{-/-}$mice exhibit only mild growth retardation and normal responses to glucose and insulin (19). Therefore, in the study of SOCS molecules, the knockout approach has consistently been the most stringent assay of gene function.

Strain-dependent phenotypes in Socs $7^{-1-}$ mice. Approximately half of Socs $7^{-1-}$ mice on a C57BL genetic background exhibit hydrocephalus, growth retardation, and hypoglycemia associated with death within several weeks of birth. Krebs et al. describe a similar phenotype in independently generated Socs7-deficient mice on a pure C57BL strain (29). Their detailed histological analysis revealed 
developmental defects in the subcommissural organ, which may result in hydrocephalus.

While further experiments are required to determine the cause of the developmental defects in the subcommissural organ, it is possible that this phenotype is caused by dysregulated insulin and Igf- 1 signaling. One report describes a correlation between idiopathic perinatal hyperinsulinemia and hydrocephalus in humans (39). In mice, transgenic overexpression of Igf-binding protein-1 also results in severe hydrocephalus (40). Furthermore, Igfreceptor-1/Insr doubleknockout mice exhibited generalized subcutaneous edema (41). The absence of the hydrocephalus phenotype in half the C57BL mice and all the mixed background mice suggest that unknown modifiers of Socs7 exist to limit the penetrance of the hydrocephalus. These modifiers may be identifiable via genetic approaches.

In this study, we demonstrate significant differences in Socs7 mRNA expression levels between wild-type 129 S6 and C57BL mice in several tissues, with the greatest expression disparity being observed in isolated islets of Langerhans. These 2 inbred strains of laboratory mice differ in their susceptibility to obesity, insulin resistance, and diabetes $(42,43)$. The genetic basis for these differences has been studied in genome-wide linkage scans. In one such study, the locus corresponding to the Socs 7 gene, distal murine chromosome 11, was assigned a logarithm of the odds of linkage (LOD) score of 3.0 for hyperinsulinemia resulting from 6 weeks on a high-fat diet (44). As this is a gene-rich area of the chromosome, these results do not necessarily exclude other genes from contributing to this phenotype. We find it suggests, however, that the lower levels of Socs 7 in the islets of 129S6 mice might contribute to improved insulin signaling and sensitivity.

Regulation of islet mass in genetic studies of insulin-signaling molecules. We have demonstrated that pancreatic islets from wild-type C57BL mice express high levels of Socs 7 mRNA. Socs $7^{-1-}$ mice are born with normal islet mass but develop islet hyperplasia with age despite exhibition of insulin sensitivity and the absence of hyperglycemia. Furthermore, our results raise several important questions and suggest a role for SOCS7 in the regulation of pancreatic islets and $\beta$ cell homeostasis. Typically, increased islet mass is associated with compensatory growth in insulin-resistant states. This compensatory growth has been shown to be dependent on expression of insulinsignaling molecules. Studies have shown that mice with $\beta$ cell-specific targeted disruption of INSR or IRS2 are born with normal islet mass that exhibits a failure to expand due to lack of responsiveness to normal growth signals over time $(45,46)$. Although increased islet mass is characteristically associated with insulin resistance, it can also be found in models of increased insulin action, such as transgenic mice expressing a constitutively activated form of Akt1 (47). We propose that islets in Socs $7^{-1-}$ mice exhibit a similar phenotype of increased growth in proportion to increased insulin signaling.

SOCS7 as a regulator of IRS function. Several of our findings suggest a role for SOCS7 in regulating IRS protein stability and function. When IRS1 is coexpressed with SOCS7 in HEK293 cells, IRS1 protein levels are reduced. This apparent degradation coincides with the cellular accumulation of ubiquitinated IRS1 when the proteasome is blocked. These data suggest that SOCS7 is sufficient for IRS1 ubiquitination and degradation. In wild-type cells, chronic insulin stimulation of MEF cells causes a reduction of IRS protein levels; this phenotype is also reversible with the inclusion of proteasome inhibitors $(2,3)$. This reduction is not observed in Socs $7^{-/-}$MEF cells, which suggests that SOCS7 is necessary for insulin-stimulated IRS1 protein degradation in this cell type. Our insulin-dependent adipocyte differentiation assay supports this conclusion. In this assay, which requires incubation with insulin for several days, we found increased differentiation, a surrogate measure of insulin action, in Socs $7^{-/-}$MEF cells. Perhaps the strongest evidence for a role in regulation of insulin signaling is the increased responsiveness of Socs $7^{-1-}$ mice to ITTs and GTTs. These experiments measure the whole-body response to insulin and glucose, and the results obtained cannot be explained by differences in body mass or insulin levels. Lastly, we find increased islet size in Socs $7^{-1-}$ mice, which may also be suggestive of increased insulin sensitivity. Together, these results suggest an essential, nonredundant role for SOCS7 in regulation of IRS1 and insulin signaling in vivo.

Role of SOCS proteins in adipocyte differentiation. Insulin signaling is essential to initiation of embryonic fibroblast differentiation into adipocytes. The evidence supporting this idea comes from gene ablation studies of IRS1 or IRS2, which exhibit partial defects in adipogenesis (31). In contrast, improved insulin signaling is reported for both Socs 1 and Socs 3 deficiency; however, increased adipogenesis is only found in Socs $1^{-/-}$cells $(23,26)$. The results of increased adipogenesis found in Socs $7^{-/-}$MEF cells are consistent with the increased insulin signaling seen in vivo. Although these results would predict an increased percentage of body fat in Socs $7^{-1-}$ mice in vivo, there is no significant difference in adiposity by 8 weeks of age. In mice 9 months of age, we detected large weight variations (> 10 grams) among littermate mice of the same genotype. We believe that these differences stem from the mixed genetic background of these mice. Furthermore, both wild-type and knockout animals on the mixed background resisted dietinduced obesity (data not shown), ostensibly due to the 129S6 component of the genetic background $(43,44)$. Hence, our failure to see an in vivo correlate to increased fibroblast differentiation is confounded by these factors. In summary, our data suggest that SOCS7 is an important regulator of insulin signaling and glucose homeostasis in mice. The definition of the salient regulatory system(s) that affect insulin may lead to greater understanding of the dysregulation present in type 2 diabetes.

\section{Methods}

Generation and identification of mutant mice. A 129X1/SvJ (formerly 129/SvJ) mouse genomic library (Stratagene) was screened with a mouse Socs 7 expressed sequence tag (GenBank accession number 6256472) BamHI fragment as a probe. Exon/intron boundaries were determined by restriction enzyme mapping. A $17-\mathrm{kb}$ NotI-NotI fragment of a positive clone was subcloned into pBluescript. To generate the targeting construct, a 9-kb HindIII fragment containing 5' Socs7 genomic DNA and a 1.3-kb SmaI$\mathrm{XbaI}$ fragment containing 3 ' sequence were first cloned into pBluescript (Stratagene) and then into EcoRI/KpnI sites and XhoI/NotI sites of pPNT, respectively. The targeting construct was linearized with NotI and electroporated into CJ7 ES cells. Transfectants were selected in G418 $(300 \mu \mathrm{g} / \mathrm{ml})$ and ganciclovir $(2 \mu \mathrm{M})$. Drug resistant-colonies were analyzed for homologous recombination by Southern blot following digestion with BglII and hybridized with a $3^{\prime}$ probe (Figure 1A). From a total of 192 clones, we obtained 6 that were correctly recombined. Chimeric mice were generated by microinjection of $2 \mathrm{ES}$ cell clones into E3.5 C57BL/6J blastocysts, then transferred to pseudopregnant foster mothers. Chimeric males were mated with $\mathrm{C} 57 \mathrm{BL} / 6 \mathrm{~J}$ females, and germline transmission of the mutated allele was verified by Southern blot tail DNA from agouti-colored F1 offspring. Routine genotyping was determined by PCR. The sense primers derived from the $\operatorname{Socs} 7$ gene were $5^{\prime}$-CCAGCTCCAGGAGACTTAACA- 3 ' and $5^{\prime}$ CTGGTACGAGACAGCTCTGAT-3'. The antisense primer derived from 
the $\mathrm{Neo}^{\mathrm{R}}$ gene was $5^{\prime}$-CTACCGGTGGATGTGGAATGT- ${ }^{\prime}$. The reaction was started at $94^{\circ} \mathrm{C}$ and proceeded for 2 minutes. This was followed by 35 cycles at $94^{\circ} \mathrm{C}$ ( 30 seconds), $58^{\circ} \mathrm{C}\left(30\right.$ seconds), and $72^{\circ} \mathrm{C}$ ( 45 seconds). The wild-type allele resulted in a $250-\mathrm{bp}$ band and the recombinant allele in a 320-bp band. All experimental mouse protocols adhered to Institutional Animal Care and Use Committee (IACUC) guidelines and were approved by the IACUC of Columbia University Medical Center.

Blood glucose, plasma insulin, and glucagon concentrations. Prior to measuring basal blood glucose and plasma insulin concentrations, we fasted 6- to 8week-old mice overnight (12-16 hours). Blood was obtained by retro-orbital bleeds on unanesthetized mice. Blood glucose levels were measured using an automatic glucometer (Roche Diagnostics Corp.). Plasma insulin and glucagon levels were measured by radioimmunoassay (Linco Research Inc.).

GTT and ITT. GTT was performed as described. After an overnight fast, mice were injected intraperitoneally with $2 \mathrm{~g} / \mathrm{kg}$ body weight of D-glucose. Plasma glucose levels were determined from the mouse tail vein at 0,30 , 60,90 , and 120 minutes after injection. ITT was performed as previously described $(30,31)$, using an intraperitoneal injection of human insulin ( $0.75 \mathrm{U} / \mathrm{kg}$ body weight) in unanesthetized mice. Thereafter, blood was drawn from the tail vein at $0,20,40,60$, and 120 minutes, and plasma glucose levels were measured.

Northern analysis and Q-PCR. Random fed female mice aged 4-6 weeks from the Jackson Laboratory (C57BL/6J) or Taconic (129S6SvEv) were either unperturbed or treated with a $0.75-\mathrm{U} / \mathrm{kg}$ intraperitoneal bolus of insulin. Total RNA was extracted from tissues using TRIzol (Invitrogen Corp.) according to the manufacturer's protocol. Two micrograms per lane of mRNA was fractionated by electrophoresis on a $1 \%$ agarose-formaldehyde gel, transferred to Duralose-UV nitrocellulose membrane, and fixed by UV cross-linking. The membranes were then hybridized with ${ }^{32} \mathrm{P}$-radiolabeled mouse cDNA of Socs7 or a Gapdh fragment as probes. For Q-PCR, RNA was further purified through a RNeasy column according to the manufacturer's protocol (QIAGEN). cDNA synthesis was performed with $2 \mu \mathrm{g}$ of total DNase free-RNA with Omniscript (QIAGEN) according to the manufacturer's instructions. Real-time PCR reactions were performed using QuantiTect SYBR Green PCR Kit (QIAGEN) on an ABI PRISM 7700 (Applied Biosystems). Plasmid controls for Socs 7 and Hprt were included on each plate for determination of absolute copy number. A 150-bp Socs 7 fragment was generated from the following primers: sense, 5'-TGACAAGAACTCAAAGTGCC- ${ }^{\prime}$; and antisense, $5^{\prime}$-GGCTTCTTCTTGGAGGAGGA-3'. A 150-bp Pparg fragment was generated from the following primers: sense, $5^{\prime}$-TCTGCTCAAGTATGGTGTCCA-3'; and antisense, $5^{\prime}$-AGTTCCAGGGCCTGCAGCAGG-3'. Reactions were performed at $95^{\circ} \mathrm{C}$ for 15 minutes; this was followed by 50 cycles at $94^{\circ} \mathrm{C}$ ( 15 seconds), $60^{\circ} \mathrm{C}\left(30\right.$ seconds), and $72^{\circ} \mathrm{C}(30$ seconds).

Plasmid generation and cell culture. HEK293 cells were transfected with the calcium phosphate precipitation method as described previously (48). The plasmid for the truncated form of Socs7, pGEX2T-NAP4, was a generous gift from T. Takenawa (Tokyo Metropolitan Institute of Gerontology, Tokyo, Japan), and the remaining sequence was obtained by high fidelity PCR from a human SOCS7 (hSOCS7) expressed sequence tag (I.M.A.G.E. clone number 2387107). Our full-length human Socs7 cDNA has a predicted amino acid sequence matching the GenBank sequence NP_055413, with the exception of a spontaneous mutation, P349S. This cDNA was placed in frame with the dual N-terminal His and Xpress epitope tags of pCDNA3HisA (Invitrogen Corp.). The SOCS7 mutants were generated as follows: $\Delta \mathrm{N}$ has an $\mathrm{N}$-terminal 352 -amino acid deletion; $\Delta \mathrm{SB}$ has a C-terminal 79-amino acid deletion; and the $\mathrm{R} \rightarrow \mathrm{K}$ construct substitutes R425K and S427C. For cell culture experiments shown in Figures 4C and 5A, MEF or HEK293 cells were pretreated with $20 \mu \mathrm{M}$ MG132 or $10 \mu \mathrm{M}$ lactacystin (Sigma-Aldrich) for 30 minutes before a 6-hour treatment with $10 \mathrm{nM}$ IGF-1 (R\&D Systems) or $100 \mathrm{nM}$ insulin (Lilly). Cell culture experiments for Figures 1D and 4C included no insulin other than that found in $10 \%$ serum.

Western analysis and immunoprecipitation. Human insulin $(0.75 \mathrm{U} / \mathrm{kg})$ was injected as a bolus into the inferior venae cavae or intraperitoneal cavities of anaesthetized mice. The quadriceps muscles were removed at 5 or 60 minutes after insulin injection and were frozen on dry ice. Cellular lysates were homogenized in NP-40 lysis buffer containing 0.5\% NP-40, $50 \mathrm{mM}$ Tris pH 8.0, $10 \%$ glycerol, $0.1 \mathrm{mM}$ EDTA, $150 \mathrm{mM} \mathrm{NaCl}, 0.5 \mathrm{mM} \mathrm{Na}_{3} \mathrm{VO}_{4}$, $4.5 \mathrm{mM} \mathrm{Na}_{2} \mathrm{P}_{4} \mathrm{O}_{7}, 50 \mathrm{mM} \mathrm{NaF}, 1 \mathrm{mM}$ DTT, $0.4 \mathrm{mM}$ PMSF, $3 \mu \mathrm{g} / \mathrm{ml}$ aprotinin, $1 \mu \mathrm{g} / \mathrm{ml}$ leupeptin, and $2 \mu \mathrm{g} / \mathrm{ml}$ pepstatin. The homogenates were solubilized for 1 hour at $4{ }^{\circ} \mathrm{C}$ and clarified by centrifugation at $16,000 \mathrm{~g}$ for 30 minutes. Supernatants containing equal amounts of protein (2-3 $\mathrm{mg}$ ) were immunoprecipitated ( $\geq 2$ hours or overnight) with $2 \mu \mathrm{g}$ of an antibody against INSR $\beta$ subunit (Santa Cruz Biotechnology Inc.), an antiIRS1 antibody (Upstate), or an anti-IRS2 antibody (Upstate). Immune complexes were collected with $60 \mu \mathrm{l}$ of a $50 \%$ slurry of protein A agarose (Santa Cruz) resolved on $6 \%$ SDS-PAGE and transferred to nitrocellulose. The blots were probed with antibodies against INSR $\beta$ subunit, IRS1, Xpress epitope (Invitrogen Corp.), ubiquitin (Santa Cruz Biotechnology Inc.), or antiphosphotyrosine 4G10 (Upstate) (48).

Histology. Pancreata from 6 wild-type and 6 Socs $7^{-/}$mice were fixed in formalin, sectioned, and stained with anti-insulin or antiglucagon antisera, then counterstained with an alkaline phosphatase secondary antibody. Two sections from each pancreas were imaged and quantified for surface area with SPOT Image analysis software (version 4.0; Diagnostic Instruments Inc.).

Adipocyte differentiation. MEF cells were collected from 11.5-14.5 dpc (days post coitum) embryos, seeded into 12 -well plates, and propagated to confluence. From 3 to 5 cell lines were tested for each genotype. Socs 7 -sufficient or -deficient cells were developed from C57BL/6 embryos. After 48 hours, differentiation was induced using $5 \mu \mathrm{g} / \mathrm{ml}$ insulin, $1 \mu \mathrm{M}$ dexamethasone, $0.5 \mathrm{mM}$ IBMX, and $10 \mu \mathrm{M}$ troglitazone (Sigma-Aldrich). After 2 days, media were replaced with a maintenance medium of insulin and troglitazone. After 14 days of differentiation, lipid drops in the cells were stained with oil red $\mathrm{O}$, or RNA was extracted as described above. 3T3-L1 cells were differentiated as previously described (5).

Analysis. Statistical analyses were performed with Microsoft Excel software using 1-tailed Student's $t$ tests. A $P$ value less than 0.05 was considered significant.

\section{Acknowledgments}

We would like to thank Marija Chouinard and Rudy Leibel for the generous gift of isolated islet mRNA and Anne-Marie Brillantes for help with islet morphometry and surface area quantification. This work was supported in part by NIH grant IP30-DK63068.

Received for publication November 8, 2004, and accepted in revised form June 14, 2005.

Address correspondence to: Paul B. Rothman, Department of Internal Medicine, SE308 GH, University of Iowa, Roy J. and Lucille A. Carver College of Medicine, 200 Hawkins Drive, Iowa City, Iowa 52242, USA. Phone: (319) 384-5424; Fax: (319) 356-8608; E-mail: paul-rothman@uiowa.edu.

Alexander S. Banks and Jianze Li contributed equally to this work.

Note added in proof. A recent paper describing enhanced hepatic insulin signaling in Socs $1^{-/-} \mathrm{Ifng}^{-/-}$mice has helped to further clarify the differential roles of SOCS molecules in this pathway (49). 
1. Rice, K.M., Turnbow, M.A., and Garner, C.W. 1993. Insulin stimulates the degradation of IRS- 1 in 3T3-L1 adipocytes. Biochem. Biophys. Res. Commun. 190:961-967.

2. Zhande, R., Mitchell, J.J., Wu, J., and Sun, X.J. 2002. Molecular mechanism of insulin-induced degradation of insulin receptor substrate $1 . \mathrm{Mol}$. Cell. Biol. 22:1016-1026.

3. Sun, X.J., Goldberg, J.L., Qiao, L.Y., and Mitchell, J.J. 1999. Insulin-induced insulin receptor substrate-1 degradation is mediated by the proteasome degradation pathway. Diabetes. 48:1359-1364.

4. Senn, J.J., Klover, P.J., Nowak, I.A., and Mooney, R.A. 2002. Interleukin-6 induces cellular insulin resistance in hepatocytes. Diabetes. 51:3391-3399.

5. Rui, L., Fisher, T.L., Thomas, J., and White, M.F. 2001. Regulation of insulin/insulin-like growth factor-1 signaling by proteasome-mediated degradation of insulin receptor substrate-2. J. Biol. Chem. 276:40362-40367.

6. Rui, L., Yuan, M., Frantz, D., Shoelson, S., and White, M.F. 2002. SOCS-1 and SOCS-3 block insulin signaling by ubiquitin-mediated degradation of IRS1 and IRS2. J. Biol. Chem. 277:42394-42398.

7. Haruta, T., et al. 2000. A rapamycin-sensitive pathway down-regulates insulin signaling via phosphorylation and proteasomal degradation of insulin receptor substrate-1. Mol. Endocrinol. 14:783-794.

8. Saltiel, A.R., and Kahn, C.R. 2001. Insulin signalling and the regulation of glucose and lipid metabolism. Nature. 414:799-806.

9. Hotamisligil, G.S., et al. 1996. IRS-1-mediated inhibition of insulin receptor tyrosine kinase activity in TNF-alpha- and obesity-induced insulin resistance. Science. 271:665-668.

10. Aguirre, V., Uchida, T., Yenush, L., Davis, R., and White, M.F. 2000. The c-Jun NH(2)-terminal kinase promotes insulin resistance during association with insulin receptor substrate- 1 and phosphorylation of Ser(307). J. Biol. Chem. 275:9047-9054.

11. Gao, Z., et al. 2002. Serine phosphorylation of insulin receptor substrate 1 by inhibitor kappa B kinase complex. J. Biol. Chem. 277:48115-48121.

12. Paz, K., et al. 1997. A molecular basis for insulin resistance. Elevated serine/threonine phosphorylation of IRS- 1 and IRS- 2 inhibits their binding to the juxtamembrane region of the insulin receptor and impairs their ability to undergo insulininduced tyrosine phosphorylation. J. Biol. Chem. 272:29911-29918.

13. Chen, X.P., Losman, J.A., and Rothman, P. 2000. SOCS proteins, regulators of intracellular signaling. Immunity. 13:287-290.

14. Johnston, A.M., Pirola, L., and Van Obberghen, E. 2003. Molecular mechanisms of insulin receptor substrate protein-mediated modulation of insulin signalling. FEBS Lett. 546:32-36.

15. Krebs, D.L., and Hilton, D.J. 2003. A new role for SOCS in insulin action. Suppressor of cytokine signaling [review]. Sci. STKE. 2003:PE6.

16. Kile, B.T., et al. 2002. The SOCS box: a tale of destruction and degradation. Trends Biochem. Sci. 27:235-241.

17. Matuoka, K., Miki, H., Takahashi, K., and Takenawa, T. 1997. A novel ligand for an SH3 domain of the adaptor protein Nck bears an $\mathrm{SH} 2$ domain and nuclear signaling motifs. Biochem. Biophys. Res.
Commun. 239:488-492.

18. Martens, N., et al. 2004. The suppressor of cytokine signaling (SOCS)-7 interacts with the actin cytoskeleton through vinexin. Exp. Cell Res. 298:239-248.

19. Krebs, D.L., et al. 2002. SOCS-6 binds to insulin receptor substrate 4 , and mice lacking the SOCS-6 gene exhibit mild growth retardation. Mol. Cell. Biol. 22:4567-4578.

20. Peraldi, P., Filloux, C., Emanuelli, B., Hilton, D.J., and Van Obberghen, E. 2001. Insulin induces suppressor of cytokine signaling-3 tyrosine phosphorylation through janus-activated kinase. J. Biol. Chem. 276:24614-24620.

21. Emanuelli, B., et al. 2000. SOCS-3 is an insulininduced negative regulator of insulin signaling. J. Biol. Chem. 275:15985-15991.

22. Emanuelli, B., et al. 2001. SOCS-3 inhibits insulin signaling and is up-regulated in response to tumor necrosis factor-alpha in the adipose tissue of obese mice. J. Biol. Chem. 276:47944-47949.

23. Kawazoe, Y., et al. 2001. Signal transducer and activator of transcription (STAT)-induced STAT inhibitor 1 (SSI-1)/suppressor of cytokine signaling 1 (SOCS1) inhibits insulin signal transduction pathway through modulating insulin receptor substrate 1 (IRS-1) phosphorylation. J. Exp. Med. 193:263-269.

24. Mooney, R.A., et al. 2001. Suppressors of cytokine signaling- 1 and -6 associate with and inhibit the insulin receptor. A potential mechanism for cytokine-mediated insulin resistance. J. Biol. Chem. 276:25889-25893.

25. Ueki, K., Kondo, T., and Kahn, C.R. 2004. Suppressor of cytokine signaling 1 (SOCS-1) and SOCS-3 cause insulin resistance through inhibition of tyrosine phosphorylation of insulin receptor substrate proteins by discrete mechanisms. Mol. Cell. Biol. 24:5434-5446.

26. Shi, H., Tzameli, I., Bjorbaek, C., and Flier, J.S. 2004. Suppressor of cytokine signaling 3 is a physiological regulator of adipocyte insulin signaling. J. Biol. Chem. 279:34733-34740.

27. Rawlings, J.S., Rennebeck, G., Harrison, S.M., Xi, R., and Harrison, D.A. 2004. Two Drosophila suppressors of cytokine signaling (SOCS) differentially regulate JAK and EGFR pathway activities. BMC Cell Biol. 5:38. doi:10.1186/1471-2121-5-38.

28. Li, L., et al. 2004. Insulin induces SOCS-6 expression and its binding to the p 85 monomer of phosphoinositide 3-kinase, resulting in improvement in glucose metabolism. J. Biol. Chem. 279:34107-34114.

29. Krebs, D.L., et al. 2004. Development of hydrocephalus in mice lacking SOCS7. Proc. Natl. Acad. Sci. U. S. A. 101:15446-15451.

30. Sadowski, C.L., et al. 2001. Insulin induction of SOCS-2 and SOCS-3 mRNA expression in C2C12 skeletal muscle cells is mediated by Stat 5 * J. Biol. Chem. 276:20703-20710.

31. Miki, H., et al. 2001. Essential role of insulin receptor substrate 1 (IRS-1) and IRS-2 in adipocyte differentiation. Mol. Cell. Biol. 21:2521-2532.

32. Peng, X.D., et al. 2003. Dwarfism, impaired skin development, skeletal muscle atrophy, delayed bone development, and impeded adipogenesis in mice lacking Akt1 and Akt2. Genes Dev. 17:1352-1365.

33. Nakae, J., et al. 2003. The forkhead transcription factor Foxo1 regulates adipocyte differentiation.
Dev. Cell. 4:119-129.

34. Magun, R., et al. 1996. Expression of a constitutively activated form of protein kinase B (c-Akt) in 3T3-L1 preadipose cells causes spontaneous differentiation. Endocrinology. 137:3590-3593.

35. Goodyear, L.J., et al. 1995. Insulin receptor phosphorylation, insulin receptor substrate-1 phosphorylation, and phosphatidylinositol 3-kinase activity are decreased in intact skeletal muscle strips from obese subjects. J. Clin. Invest. 95:2195-2204.

36. Rondinone, C.M., et al. 1997. Insulin receptor substrate (IRS) 1 is reduced and IRS- 2 is the main docking protein for phosphatidylinositol 3-kinase in adipocytes from subjects with non-insulindependent diabetes mellitus. Proc. Natl. Acad. Sci. U. S. A. 94:4171-4175.

37. Alexander, W.S., and Hilton, D.J. 2004. The role of suppressors of cytokine signaling (SOCS) proteins in regulation of the immune response. Annu. Rev. Immunol. 22:503-529.

38. Mauvais-Jarvis, F., et al. 2002. Reduced expression of the murine p85alpha subunit of phosphoinositide 3-kinase improves insulin signaling and ameliorates diabetes. J. Clin. Invest. 109:141-149. doi:10.1172/JCI200213305.

39. de Lonlay, P., et al. 2002. Facial appearance in persistent hyperinsulinemic hypoglycemia. Am. J. Med. Genet. 111:130-133.

40. Doublier, S., Duyckaerts, C., Seurin, D., and Binoux, M. 2000. Impaired brain development and hydrocephalus in a line of transgenic mice with liver-specific expression of human insulin-like growth factor binding protein-1. Growth Horm. IGF Res. 10:267-274.

41. Louvi, A., Accili, D., and Efstratiadis, A. 1997. Growth-promoting interaction of IGF-II with the insulin receptor during mouse embryonic development. Dev. Biol. 189:33-48.

42. West, D.B., Boozer, C.N., Moody, D.L., and Atkinson, R.L. 1992. Dietary obesity in nine inbred mouse strains. Am. J. Physiol. 262:R1025-R1032.

43. Goren, H.J., Kulkarni, R.N., and Kahn, C.R. 2004. Glucose homeostasis and tissue transcript content of insulin signaling intermediates in four inbred strains of mice: C57BL/6, C57BLKS/6, DBA/2, and 129X1. Endocrinology. 145:3307-3323.

44. Almind, K., and Kahn, C.R. 2004. Genetic determinants of energy expenditure and insulin resistance in diet-induced obesity in mice. Diabetes. 53:3274-3285.

45. Kubota, N., et al. 2004. Insulin receptor substrate 2 plays a crucial role in $\beta$ cells and the hypothalamus. J. Clin. Invest. 114:917-927. doi:10.1172/ JCI200421484.

46. Kulkarni, R.N., et al. 1999. Tissue-specific knockout of the insulin receptor in pancreatic beta cells creates an insulin secretory defect similar to that in type 2 diabetes. Cell. 96:329-339.

47. Tuttle, R.L., et al. 2001. Regulation of pancreatic beta-cell growth and survival by the serine/threonine protein kinase Akt1/PKBalpha. Nat. Med. 7:1133-1137.

48. Limnander, A., Danial, N.N., and Rothman, P.B. 2004. v-Abl signaling disrupts SOCS-1 function in transformed pre-B cells. Mol. Cell. 15:329-341.

49. Jamieson, E., et al. 2005. SOCS1 deficiency enhances hepatic insulin signaling. J. Biol. Chem. In press. 